\title{
Body language reading of emotion and outcome in schizophrenia
}

Egeland, Jens ${ }^{1,2}$; Holmen, Tom Langerud ${ }^{1}$, Bang-Kittilsen, Gry ${ }^{1}$, Bigseth, Therese Torgersen ${ }^{1}$, Vaskinn, Anja. ${ }^{3,4}$, Engh, John Abel ${ }^{1}$

Affiliation:

${ }^{1}$ Division of Mental Health \& Addiction, Vestfold Hospital Trust, Norway

${ }^{2}$ Department of Psychology, University of Oslo, Norway

${ }^{3}$ NORMENT KG Jebsen Centre for Psychosis Research, Oslo University Hospital, Norway

${ }^{4}$ Institute of Clinical Medicine, University of Oslo, Norway

Address correspondence to:

Jens Egeland

Research Unit,

Division of Mental Health \& Addiction, Vestfold Hospital Trust

Box 2267

N-3103 Tønsberg, Norway

Phone: +47 90891121

E-mail: jens.egeland@siv.no 


\begin{abstract}
:
Introduction:

Although considered a promising design for testing social cognition, it is not clear to what extent the EmoBio test of emotion recognition actually predicts community functioning. It is also not clear whether the test measures something unique or different from nonsocial cognition. The present study tests whether EmoBio accounts for GAF function score and two operationalized community outcome measures. The study also analyzes cognitive predictors of EmoBio performance, testing whether stimulus modality affects prediction.
\end{abstract}

\title{
Methods:
}

Eighty-three patients with schizophrenia were tested with EmoBio, the cognitive battery MCCB and WAIS-IV.

\section{Results:}

EmoBio accounted for a significant portion of variance in all three outcome measures. Only EmoBio predicted Lifetime Relationship status, and EmoBio remained a significant predictor of Independent living beyond non-social cognition. All cognitive measures contributed significantly to the variance in EmoBio, but entered together explained only a third of total variance.

\section{Conclusion:}

The study shows that emotion recognition accounts for community outcome. There was no clear effect of test-modality in predicting EmoBio performance, indicating no method invariance problem with EmoBio. It also indicates that the mechanisms underlying impaired social cognition in schizophrenia are different from the hypothesized non-verbal learning deficits in the disorder.

Keywords: EmoBio, social cognition, schizophrenia, outcome

Being able to understand and interpret other persons' emotions, intentions and actions, often referred to as social cognition, is important for successful interactions with others, and thus for being able to establish good and stable relations as well as for vocational achievements (Fett, Viechtbauer, Dominguez, Penn, van Os, \& Krabbendam, 2011). Subjects with schizophrenia are impaired in social cognition (Savla et al. 2013). Research through several decades has focused on the cognitive deficits related to the illness as a possible cause of the impaired social function (Green,1996). However, more recent evidence points to the usefulness of a differentiation between social and non-social cognition, and that social 
EmoBio and outcome

cognition acts as a mediator between non-social cognition and community functioning (Schmidt, Mueller \& Roder, 2011; Green, Lee \& Ochsner, 2013).

One methodology that has been used to study social cognition in schizophrenia are point-light displays (PLDs). In this type of stimuli, a light is connected to an object or parts of the human body that move in a dark room. Thus, all information except that of the motion is eliminated, enabling investigation of pure motion, devoid of shape or color. Such tasks can be non-social, as when a ball rotates or a saw is in action, or they can demand social interpretation. In the latter case, one can differentiate between interpreting actions, such as climbing or digging, or emotional states, such as anger or happiness. A somewhat related distinction is between non-biological and biological motion. The rotating ball represents nonbiological motion, while a person moving is biological motion, but does not necessitate a social or emotional interpretation. In a recent meta-study of the 15 PLD studies that have been conducted so far in schizophrenia, Okruszek and Pilecka (2017) found an effect size of 0.61 for emotion recognition when comparing healthy controls to patients with schizophrenia. Okruszek and Pilecka claim that initially in this strain of studies, researchers reported a dissociation between biological motion processing, which was impaired, and form perception, which was intact. This is similar to what is found in autism, i.e. a specific deficit in interpreting (emotional) biological motion, with less impairment in the interpretation of nonhuman objects (Hubert et al., 2007; Nackaerts, Wagemans, Helsen, Swinnen, Wenderoth, \& Alaerts, 2012). In the same vein, Lee et al. (2013) found a dissociation between patients with schizophrenia and bipolar disorder. The schizophrenia group was more impaired in social relative to nonsocial cognitive domains, while the opposite was found among patients with bipolar disorder. According to Okruszek and Pilecka (2017) later studies have questioned the claim of a particular impairment in interpreting social stimuli vs non-social motion, and instead pointed to impairment already in lower level visual abilities (Brittain, ffytche, 
McKendrick and Surguladze, 2010; Kim, Norton, McBain, Ongur, \& Chen, 2013) as well as correlations to general cognition (Olbert et al., 2013). Thus, it is not settled whether the impairment in processing biological motion and emotional biological motion specifically, is a genuine social interpretation impairment. I could also be secondary to specific cognitive difficulties, whether in visual processes or in general cognition.

In schizophrenia research, the PLD methodology has been used mainly for biological motion, and particularly for emotional biological motion. Six of the 15 studies cited by Okruszek and Pilecka (2017) applied the Emotion in Biological Motion PLD test (EmoBio: Heberlein, Adolphs, Tranel, \& Damasio, 2004). This test was among several measures from the social neuroscience field, with known links to specific neural systems, that were selected by the Social Cognition and Functioning in Schizophrenia (SCAF) study (Green et al., 2013). The goal of the SCAF study was to examine if measures from social neuroscience could be adapted to schizophrenia clinical trials. The EmoBio test was considered a valid instrument of nonverbal social cognition. It was found to be well tolerated and able to distinguish between individuals with schizophrenia and healthy controls (Kern et al, 2013). The external validity of the EmoBio test was considered to be modest, and further refinement was therefore recommended before use in clinical trials (Olbert et al, 2013). The SCAF program rests on the premise that social cognition is a stronger predictor of outcome than non-social cognition (Green et al., 3013). Testing predictions from four different social cognition tasks, among them the EmoBio test, Olbert and colleagues (2013) nevertheless found that the associations were disappointingly low. The EmoBio test did not have incremental external validity in explaining functional outcome beyond the effect of non-social cognition. Associations were particularly low between social cognition and self-reported community functioning. Similarly, Vaskinn et al (2016) reported a non-significant relation between EmoBio and outcome, measured by the Global Assessment of Functioning (GAF-f: Aas, 2011). In a follow-up study 
using a partly overlapping sample, Engelstad, Sundet, Andreassen and Vaskinn (2017) found that EmoBio predicted social problem-solving skills assessed in a role-play, but not selfreported community outcome. It is line with the SCAF study (Olbert et al, 2013), which showed significant associations with similar measures of functional capacity, but not with community outcome.

The first research aim of the present study, is to test the predictive power of non-social cognition and EmoBio on three measures of outcome: The first measure analyzed is the function scale of the split Global Assessment of Functioning (GAF-f: Aas, 2011), replicating Vaskinn et al (2016). Further, we expand the previous literature, which focused on selfreported community outcome (Olbert et al, 2013; Engelstad et al, 2017), by applying two measures of community outcome that are precisely operationalized, with little room for subjective interpretation. We hypothesize that both non-social cognition and EmoBio will predict daily life functioning, assessed objectively. Further, we expect EmoBio to have incremental validity, i.e. will predict variance in functioning beyond the effect of non-social cognition. Three types of non-social cognition will be examined: Intelligence, attention/processing speed and learning. Based on previous research that found learning to be an especially strong predictor of functioning (Green, 1996), we separated the two learning tests from the other non-social cognitive tests in our neuropsychological battery, the Matrics Consensus Cognitive Battery (MCCB: Nuechterlein et al., 2008) In addition, the contribution of verbal- and visual-perceptual intelligence will be examined. We ask whether EmoBio contributes uniquely to the prediction; whether tests of verbal and visual-perceptual intelligence predict equally well; and whether they share variance with EmoBio in predicting outcome. 
Expecting to find that EmoBio predicts daily life functioning, an important question is what predicts EmoBio performance? This is our second research aim. The null-hypothesis would be that it correlates highly with IQ, i.e. global cognition. An alternative hypothesis, since it is a visually presented test, is that EmoBio performance is dependent upon visualperceptual intelligence specifically, over and above a general effect of global cognition. It is possible that the processing of movie clips correlates with performance on other visually presented tasks, merely by taxing modality specific processing capacity rather than depend on intelligence in general. The studies by Brittain et al (2010) and Kim et al (2013) indicate a special role for visual non-social cognition for interpreting social stimuli. Thus, we will first test the predictive power of verbal and visual-perceptual intelligence, and verbal and visual learning separately. Those that contribute significantly will subsequently be entered simultaneously in a new analysis. With this approach, we should be able to tease out the unique and shared contribution of modality (visual versus verbal processes). If modality matters, the visual measures should not only predict EmoBio performance, but account for unique variance.

Summing up the hypotheses: In our first research aim, we expect that both social and non-social cognition will contribute to functional outcome, but that social cognition (EmoBio) will be a stronger predictor. In our second research aim, we hypothesize that non-social cognition will account for a significant portion of the variance in social cognition (EmoBio performance), but that visual non-social cognition (visual-perceptual intelligence, visual learning) will account for more variance than verbal non-social cognition (verbal intelligence, verbal learning).

\section{Methods}

Participants 
EmoBio and outcome

Eighty-three participants with a Diagnostic and Statistical Manual of Mental Disorders (American Psychiatric Association, 2013) schizophrenia spectrum disorder (schizophrenia $=62$, schizoaffective disorder=16), took part in the study. They were recruited from the baseline assessment of the randomized, controlled, observer-blinded clinical trial 'Effects of Physical Activity in Psychosis' (EPHAPS) (ClinicalTrials.gov, registration number NCT02205684; 37).

The participants were recruited from August 2014 through February 2017 from catchment area-based and publicly funded outpatient psychiatric clinics in Vestfold County, Norway. A subgroup of patients was referred from primary health care to the outpatient clinics for participation in the project specifically. Diagnosis was established using the Structured Clinical Interview for Diagnostic and Statistical Manual of Mental Disorders (DSM-V) axis I disorders (SCID I: First, Spitzer, Gibbon, \& Williams, 2002). Interviews were conducted by a clinical psychologist or a specialist in psychiatry. Members of the assessment staff attended a course based on the SCID training program at the University of California Los Angeles (Ventura, Liberman, Green, Shaner, \& Mintz, 1998). All participated in diagnostic consensus meetings.

Additional inclusion criteria were: age between 18 and 67; understanding and speaking a Scandinavian language. Exclusion criteria were: pregnancy; chest pain during CRF test; unstable angina pectoris; recent myocardial infarction; uncontrollable cardiac arrhythmia; severe hypertension (>180/110 mmHg); co-morbid diagnosis of mild mental retardation; and/or other medical conditions incompatible with participation. The study was approved by the Regional Committee for Medical and Health Research Ethics of Southern and Eastern Norway (file number 2014/372/REK SOER-OEST). For more details on the inclusion procedure, see Engh et al. (2015). 
EmoBio and outcome

Demographic and clinical characteristics are presented in Table 1.Eighty participants received antipsychotic medical treatment. Participants' defined daily doses (DDD) were calculated in accordance with guidelines from the World Health Organization Collaborating Center for Drug Statistics Methodology (http://www.whocc.no/atcdd). Some participants received additional anxiolytic and/or antidepressant or mood stabilizing medication $(n=21,15$ and 9, respectively). Six participants were presently married, four were cohabiting and 12 reported to previously having been married or cohabiting. The remaining sixty had always lived alone. Fifty-eight persons lived independently or together with parents, while 22 persons lived in communal supported housing and received help from communal health personnel. This information given by the subjects themselves were corroborated by patient file information.

\section{Insert table 1 about here.}

Instruments and measures

The EmoBio test is comprised of 22 short clips of point-light display walkers. The clips were shown on a computer screen, and participants indicated on a sheet of paper which emotion was displayed. Emotions were angry, happy, sad, fearful, or neutral/no emotion. We used the same proportional scoring method as previous studies (Heberlein et al.,2004); Couture et al., 2010; Vaskinn et al, 2016). Some of the clips are easy to interpret while others are more ambiguous. A response is given credit based on the proportion of healthy control participants giving that response. To be able to compare scores across studies, we applied the Norwegian norms developed from a reference group of 101 healthy control participants, randomly selected from national statistical records, by Vaskinn et al. (2016)

Three measures were used to measure psychosocial outcome: 
In addition to the examiner-rated Global Assessment of Functioning (GAF-f: Aas, 2011) we constructed two precisely operationalized measures of community functioning: The first was a classification of "lifetime relationship status", i.e. whether the subjects had always lived alone or ever in relationship. Being able to have age-appropriate involvement in intimate relationships is listed as typical of superior social/interpersonal functioning in the Global Functioning: Social scale by Cornblatt et al (2007). Although intimate relations are not limited to marriage or cohabitation, ever having lived together in an intimate relation with another person (even though presently divorced or widowed) compared to always having lived alone, has the advantage of being less subjected to subjective interpretations and could serve as a rough indication of ability to form intimate relations. As age is expected to be the foremost predictor of lifetime relationship status and the participants varied from 18 to 67 years of age, we entered age into this regression analysis.

In the Supported living outcome measure, participants were classified according to whether they at study inclusion lived independently or in supported housing. Ability to handle homemaking tasks is listed as indicative of good role functioning in Cornblatt's Global Functioning: Role scale (Cornblatt et al., 2007).

All participants were examined with the three subtests of the Wechsler Adult Intelligence Scale, version 4 (Wechsler, 2008) comprising the Verbal Comprehension Index (WAIS-VCI) and the three tests forming the Perceptual Reasoning indexes (WAIS-PRI). Together they comprise the General Ability Index (WAIS-GAI), equivalent of full scale IQ. WAIS-VCI is our measure of verbal intelligence, whereas WAIS-PRI is our measure of visual-perceptual intelligence.

The participants were also tested with the nine non-social cognitive subtests in the MCCB (Nuechterlein et al., 2008). Of these, the Hopkins Verbal Learning Test-revised 
EmoBio and outcome

(HVLT: Brandt \& Benedict, 2001) and the Brief Visual-spatial Memory Test-revised (BVMT-R: Benedict, 1997) constituted a Memory factor in the three-factor model for MCCB by Burton, Vella, Harvey, Patterson, Heaton and Twamley (2013). This is our measure of Learning (MCCB-L). Although they differ in modality, the two tests are similar in design. The subjects are presented with a list of 12 words or 6 geometric designs, respectively, and are asked to immediately report or draw the presented stimuli. Both tests have three successive acquisition trials, with points or scores awarded varying from 0 to 12 in both tests. In the present study we apply the age and gender corrected t-scores for all three acquisition trials, collapsed. HVLT is our measure of verbal learning (MCCB-verL), whereas BVMT-R is our measure of visual learning (MCCB-visL). The seven remaining MCCB tests (Trail Making Test, Symbol Coding, Spatial Span, Letter-Number-Span, Mazes, Word Fluency and the Identical pairs Continuous Performance Test) were found to constitute an attention and a psychomotor factor in the Burton et al (2013) study. Together, these tests make up our attention/speed measure (MCCB-AS). This division of the MCCB tests was made to enable us to analyze whether learning contributes more to daily life function than other non-social cognitive measures (Green, 1996).

Data analyses

The results were analyzed with series of hierarchic linear regression analyses applying SPSS version 23. In the first series of analyses, investigating our first research aim, WAISGAI, MCCB-AS, MCCB-L, and EmoBio score were entered with each of the three outcome measures as the dependent variables in three separate regression analyses. Then, in a new set of analyses, all variables that were significantly related to the outcome measures were entered simultaneously in one block in order to determine shared and unique explanatory power. Beta-values for each predictor and the p-value for their unique contribution, as well as $\mathrm{r}^{2}$ 
EmoBio and outcome

change and F-change for the last predictor added, are listed in the tables. Likewise, for our second research aim, in the second set of analyses, examining predictors of EmoBio performance, WAIS-VCI, WAIS-PRI, MCCB-AS, MCCB-verL and MCCBvisL were entered as dependent variables and EmoBio as independent variable followed by entering all significant predictors in one block.

\section{Results}

Insert Table 2 about here

Applying the norms of Vaskinn et al (2016) giving credit according to the proportion of healthy persons giving a particular response, the total EmoBio score was $.77($ s.d. $=0.15)$ which is equivalent to the findings by Engelstad et al. (2017).

The results of the three regression analyses examining our first research aim are presented in Table 2. As expected, EmoBio predicted a significant part of the variance in all three outcome measures. MCCB-AS and MCCB-L were significantly related to GAF-f. When entering all significant measures simultaneously, only MCCB-L remained significantly related to GAF-f, while the contribution of MCCB-AS and EmoBio became insignificant. For lifetime relationships only EmoBio explained significantly beyond the effect of age. For Supported living all cognitive measures explained significant portions of the variance. When entered together only MCCB-L and EmoBio remained with significant unique variance.

Insert Table 3 about here

The results of the regression analyses for our second research aim are presented in Table 3. Both WAIS-VCI, WAIS-PRI, MCCB-AS, MCCB-verL and MCCB-visL explained a 
significant portion of the variance in EmoBio. Only WAIS-PRI remained significant when entering all five predictors simultaneously in the final regression analysis.

\section{Discussion}

As expected, EmoBio scores were related to all three measures of outcome. Non-social cognition did also predict outcome, but none of the three non-social cognitive measures predicted all three outcome measures. Learning (MCCB-L) and Attention/speed (MCCB-AS) predicted GAF-f and Supported Living, while intelligence (WAIS-GAI) predicted only Supported Living. The expectation that EmoBio would predict outcome beyond non-social cognition was partly met, in the sense that it remained significant for Supported Living beyond the effect of intelligence, attention/speed and learning For Lifetime Relationship status, only EmoBio was significantly related. Entering all significant predictors for GAF-f, rendered EmoBio insignificant while learning remained significant beyond the effect of attention/speed and EmoBio.

The regression analyses investigating the contribution to EmoBio performance show a contribution of both verbal and visual intelligence (WAIS-VFI and WAIS PRI), attention/speed as well as verbal and visual learning (MCCBverL and MCCBvisL. However, the five non-social cognitive measures together predicted less than a third of the variance in EmoBio score. This supports the notion that EmoBio cannot be reduced to non-social cognition.

Visual intelligence was the only predictor that remained significant when all significant predictors were entered simultaneously. Regarding the two modality-specific learning measures there were, however, no modality effect favoring processing within the visual modality. Thus, the hypothesis that visual non-social cognition is more important than verbal non-social cognition when it comes to explaining emotion recognition measured by the 
visual EmoBio test, was not supported. Such a finding could have indicated either a method invariance-problem, or more substantially that mechanisms similar to what is found in Nonverbal learning Disorder (Rourke, 2005) underlie also social cognition impairments in schizophrenia. However, as we found no evidence of a specific relation between the visual EmoBio test and visual non-social cognitive tests, EmoBio most likely measures social cognition as a modality-independent construct. In the NLD model (Rourke, 2005) one expects that social cognition is impaired due to a developmental effect of the specific visual-spatial impairments characterizing the disorder. The lack of modality effect indicates that social cognition in schizophrenia may be due to other mechanisms than what is found among subjects with NLD.

Taken together, the present study shows that EmoBio performance predicts outcome better than well-validated, reliable measures of non-social cognition. This is expected based on a large literature that shows the strong predictive validity of social cognition (Fett et al., 2011) and its mediating role between non-social cognition and outcome ( Scmidt, Mueller, \& Roder, 2011; Green et al., 2013). The study also shows that EmoBio is related to non-social cognition. When entering five non-social cognitive measures that separately is related to EmoBio performance, two thirds of the variance in EmoBio remains unexplained, testifying to the claim that social cognition is related to, but different from non-social cognition.

There are some limitations that have to be taken into consideration when interpreting the results of this study.

The community outcome measures are rough classifications of the dimensions in question: are the subjects able to establish deep emotional and personal attachments, and do they handle homemaking? Admittedly, cohabitation is not a prerequisite for the ability to form intimate relationships, and the standard of living and the amount of private support from extended family may vary much among subjects formally living independently. On the other 
EmoBio and outcome

hand, the measures are precisely operationalized, which gives little room for subjective and possibly distorted views on social skills. In order to draw the general conclusion that EmoBio is superior to non-social cognition in explaining outcome, other domains of community outcome should have been examined as well. However, we were unable to classify the patient's degree of employment along a meaningful dimension. None of the participants were in ordinary employment. Also, the degree to which they took part in supported employment was related to their need for custody during daytime as well as to their work capacity.

The explanatory power of visual, verbal and emotion recognition onto outcome, and of visual and verbal cognition onto emotion recognition, may be specific for this sample varying much in illness severity and duration. Fanning, Bell and Fiszdon (2012) suggests that the association between cognition and social cognition are amplified if a minimum level of cognitive resources is necessary but not sufficient for normal social cognition. Using MCCB without a distinction between modality-specific scores, they found that subjects with neurocognitive deficits also had social cognitive impairments, but that only one in four of those with intact neurocognition had impaired social cognitive skills.

Taking the limitations into account, the study show that EmoBio predicts social and functional outcome better than measures of non-social cognition. It is the first study to systematically divide aspects of non-social cognitive functioning into equivalent modalityspecific measures, finding neither evidence of a method invariance problem with EmoBio nor indication of a Nonverbal Learning Disorder-mechanism underlying emotional recognition in schizophrenia.

\section{References}

Aas, I. M. (2011). Guidelines for rating global assessment of functioning (GAF). Annals of General Psychiatry, 10(1), 2. Doi: 10.1186/1744-859X-10-2. 
Beck, A. T., Baruch, E., Balter, J. M., Steer, R. A., \& Warman, D. M. (2004). A new instrument for measuring insight: the Beck Cognitive Insight Scale. Schizophrenia research, 68(2), 319-329.

Benedict R. (1997) Brief Visuospatial Memory Test-revised. Odessa, FL: Psychological Assessment Resources, Inc.

Brandt J, Benedict R. (2001). Hopkins Verbal Learning Test-revised. Lutz, FL: Psychological Assessment Resources, Inc.

Brittain, P., ffytche, D.H., McKendrick, A., \& Surguladze, S. (2010). Visual processing, social congition and functional outcome in schizophrenia. Schizophrenia Bulletin, 178, 270-275.

Burton, C.Z., Vella, L., Harvey, P.D., Patterson, T.L., Heaton, R.K., \& Twamley, E. (2013). Factor structure of the MATRICS Consensus Cognitive Battery (MCCB) in schizophrenia. Schizophrenia Research, 156, 244-248.

Cornblatt, B.A., Auther, A.M., Niendam, T., Smith, C.W., Zinberg, J., Bearden, C.E., \& Cannon, T.D. (2007). Preliminary Findings for Two New Measures of Social and Role Functioning in the Prodromal Phase of Schizophrenia, Schizophrenia Bulletin, 33, 3, 688-702, https://doi.org/10.1093/schbul/sbm029

Couture, S. M., Penn, D. L., Losh, M., Adolphs, R., Hurley, R., \& Piven, J. (2010). Comparison of social cognitive functioning in schizophrenia and high functioning autism: more convergence than divergence. Psychological medicine, 40(04), 569-579.

Engelstad, K.N., Sunder, K.S., Andreassen, O.A., \& Vaskinn, A. (2017).Body language reading of emotion in schizophrenia: Associations with symptoms and functional outcome. Scandinavian Journal of Psychology, 58, 359-366. Doi: 10.1111/sjop.12381.

Fanning, J.R., Bell, M.D., Fiszdon, J.M. (2012) Is it possible to have impaired neurocognition but good social cognition in schizophrenia? Schizophrenia Research, 135, 168-171.

Fett AK, Viechtbauer W, Dominguez MD, Penn DL, van Os J., Krabbendam L. (2011). The relationship between neurocognition and social cognition with functional outcomes in schizophrenia: a meta-analysis. Neuroscience \& Biobehavioral Review, 35,573-588.

Fine, J. G., \& Semrud-Clikeman, M. (2013). Psychopathology of nonverbal learning disabilities. In Davis, A. (Ed). Psychopathology of childhood and adolescence: A neuropsychological approach. (pp. 163-180). New York, NY, US: Springer Publishing Co; US. 
Green, M. F. (1996). What are the functional consequences of neurocognitive deficits in schizophrenia? The American Journal of Psychiatry, 153(3), 321-30.

Green, M. F., Lee, J., \& Ochsner, K. N. (2013). Adapting social neuroscience measures for schizophrenia clinical trials, part 1: ferrying paradigms across perilous waters. Schizophrenia Bulletin, 39, 1192-1200, doi: 10.1093/schbul/sbt131.

Green MF, Harris JG, Nuechterlein KH. (2014). The MATRICS consensus cognitive battery: what we know 6 years later. American Journal of Psychiatry, 171(11),1151-4. Doi:10.1176/appi.ajp.2014.14070936.

Heberlein, A.S., Adolphs, R., Tranel, D., \& Damasio, H. (2004). Cortical Regions for Judgments of Emotions and Personality Traits from Point-light Walkers. Journal of Cognitive Neuroscience, 16 (7),1143-1158. Doi: 10.1162/0898929041920423

Hubert, B., Wicker, B., Moore, D. G., Monfardini, E., Duverger, H., Da Fonseca, D., \& Deruelle, C. (2007). Brief report: recognition of emotional and non-emotional biological motion in individuals with autistic spectrum disorders. Journal of autism and developmental disorders, 37(7), 1386-1392.

Kim,J.,Norton, D., McBain, R., Ongur, D., \& Chen, Y. (2013). Deficient biological motion perception in schizophrenia: results from a motion noise paradigm. Frontiers in Psychology, 4, article 391. Doi: 10.3389/fpsyg201300391

Lee, J., Altshuler, L., Glahn, D. C., Miklowitz, D. J., Ochsner, K., \& Green, M. F. (2013). Social and nonsocial cognition in bipolar disorder and schizophrenia: relative levels of impairment. American Journal of Psychiatry, 170(3), 334-341.

Nackaerts, E., Wagemans, J., Helsen, W., Swinnen, S. P., Wenderoth, N., \& Alaerts, K. (2012). Recognizing biological motion and emotions from point-light displays in autism spectrum disorders. PloS one, 7(9), e44473.

Nuechterlein, K. H., Green, M. F., Kern, R. S., Baade, L. E., Barch, D. M., Cohen, J. D., ... \& Goldberg, T. (2008). The MATRICS Consensus Cognitive Battery, part 1: test selection, reliability, and validity. American Journal of Psychiatry, 165(2), 203-213.

Okruszek, L. \& Pilecka, I. (2017). Biological motion processing in schizophrenia - systematic review and meta-analysis. Schizophrenia Research, Published online: march 9, http://dx.doi.org/10.1016/j.schres.2017.03.013

Olbert, C.M., Penn, D.L., Kern, R.S, Lee, J., Horan, W.P., Reise, S.P. et al. (2013). Adapting social neuroscience measures for schizophrenia clinical trials, part 3: fathoming external validity. Schizophrenia Bulletin, 39, 1211-1218. 
Riggs, S. E., Grant, P. M., Perivoliotis, D., \& Beck, A. T. (2010). Assessment of cognitive insight: a qualitative review. Schizophrenia Bulletin, 38 (2), 338-350.

Rourke, B (2005). Neuropsychology of learning deficits: Past and future. Learning Disability Quarterly, 28, 111-113.

Rund, B.R., Sundet, K., Asbjørnsen, A., Egeland, J., Landrø, N.I., Lund, A., Roness, A., Stordal, K.I., Hugdahl, K.(2006): Contrasts in neuropsychological test profiles between patients with schizophrenia and recurrent non-psychotic major depression. Acta Psychiatrica Scandinavica, 113 (4),350-359. Doi: 10.1111/j.16000447.2005.00626.x

Savla,G.N.,Vella,L.,Armstrong,C.C.,Penn,D.L.,\& Twamley,E.W. (2013).Deficits in domains of socialcognition: a meta-analysis of the empirical evidence. Schizophrenia Bulletin, 39, 979-992.doi:10.1093/schbul/sbs080

Scmidt, S.J., Mueller, D.R., Roder, V.(2011). Social cognition as a mediator variable between neurocognition and functional outcome in schizophrenia: empirical revew and new results by structural equation modeling. Schizophrenia Bulletin, 37, (Suppl 2) S41S54.

Vaskinn, A., Sundet, K., Ueland, T., Agartz, I., , Melle, I, \& Andreassen, O.A. (2013). Social cognition and clinial insight in schizophrenia and bipolar disorder. Journal of Nervous and Mental Disorders, 201, 445-451.

Vaskinn, A., Sundet, K., Østefjells, T., Nymo, K., \& Ueland, T. (2016). Reading emotions from body movement: A generalized impairment in schizophrenia. Frontiers in Psychology, 6, 2058. Doi:10.3389/fpsyg.2015.02058 
Table 1: Demographic and clinical characteristics

\begin{tabular}{|c|c|c|}
\hline Descriptives: & Mean (s.d.) & Range \\
\hline Sex M/F & $51 / 32$ & \\
\hline Age & $36(14)$ & $18-67$ \\
\hline Age at 1. Episode & $23.1(6.7)$ & $14-46$ \\
\hline Antipsychotic medication ${ }^{1}$ & $1.59(.94)$ & $0-4.5$ \\
\hline \multicolumn{3}{|l|}{ Study variables: } \\
\hline $\mathrm{VCI}^{2}$ & $89(15)$ & $60-131$ \\
\hline $\mathrm{PRI}^{3}$ & $90(15)$ & $58-124$ \\
\hline MCCB- NCS ${ }^{4}$ & $29(7)$ & $13-47$ \\
\hline $\mathrm{GAF}-\mathrm{f}^{5}$ & $44(8)$ & $23-63$ \\
\hline GAF-s ${ }^{5}$ & $43(8)$ & $23-66$ \\
\hline PANSS $^{6}$ Positive symptoms & $15(5)$ & $7-30$ \\
\hline PANSS Negative symptoms & $18(7)$ & $7-32$ \\
\hline PANSS General symptoms & $33(8)$ & $17-53$ \\
\hline EmoBio Total score & $.71(.17)$ & $.14-.97$ \\
\hline
\end{tabular}

${ }^{1}$ DDD: Defined Daily Doses.

${ }^{2}$ WAIS-IV Verbal Comprehension Index

${ }^{3}$ WAIS-IV Perceptual Reasoning Index

${ }^{4}$ Matrics Consensus Cognitive Battery- Neurocognitive composite score

${ }^{5}$ Global Assessment of Functioning- function (f) and symptoms (s) scores

${ }^{6}$ Positive and Negative Syndrome Scale 
Table 2: Predictors of outcome

\begin{tabular}{|c|c|c|c|c|}
\hline & $\mathrm{R} 2$ & Beta & $\begin{array}{c}\mathrm{R} 2 \\
\text { change }\end{array}$ & F change \\
\hline \multicolumn{5}{|c|}{ Independent measure: GAF-f: } \\
\hline WAIS-GAI $^{1}$ & .037 & .192 & & 3.066 \\
\hline${\mathrm{MCCB}-\mathrm{AS}^{2}}^{2}$ & .077 & $.277 *$ & & 6.667 \\
\hline MCCB-L ${ }^{3}$ & .130 & $.360 * *$ & & 11.903 \\
\hline EmoBio & .061 & $.246^{*}$ & & 5.171 \\
\hline MCCB-AS+ MCCB-L+ EmoBio & .146 & $.065 ; .278^{*} ; .115$ & .011 & .970 \\
\hline \multicolumn{5}{|c|}{ Independent measure:Lifetime relationship status } \\
\hline Age+ WAIS-GAI & .202 & $.457 * * ; .072$ & .005 & .498 \\
\hline Age+ MCCB-AS & .177 & $.446^{* *} ; .026$ & .001 & .067 \\
\hline Age+ MCCB-L & .219 & $.460 * * ; .148$ & .022 & 2.193 \\
\hline Age +EMOBIO & .197 & $.523 * * ; .228 *$ & .046 & .4 .765 \\
\hline \multicolumn{5}{|c|}{ Independent measure:Supported living } \\
\hline WAIS-GAI & .062 & $-.249 *$ & & 5.217 \\
\hline MCCB-AS & .070 & $-.265 *$ & & 5.963 \\
\hline MCCB-L & .127 & $.356^{* *}$ & & 11.454 \\
\hline EmoBio & .080 & $-.283 *$ & & 6.938 \\
\hline $\begin{array}{l}\text { WAIS-GAI+MCCB-AS, MCCB-L+ } \\
\text { EmoBio }\end{array}$ & .181 & $-.135 ;-.70 ;-291^{*},-.267^{*}$ & .049 & 4.534 \\
\hline
\end{tabular}

${ }^{1}$ Wechsler Adult Intelligence Scale-General Ability Index ${ }^{2}$ Matrics Consensus Cognitive Battery

(MCCB) composite measure of attention and speed. ${ }^{3} \mathrm{MCCB}$ composite measure of Learning. $* \mathrm{p}<.05, * * \mathrm{p}=<.001$ 
EmoBio and outcome

Table 3: Predictors of EMOBIO performance.

\begin{tabular}{|l|c|c|c|c|}
\hline & R2 & Beta & R2 change & F change \\
\hline WAIS- VCI $^{1}$ & .211 & $.459^{* *}$ & & 21.605 \\
\hline WAIS-PRI $^{2}$ & .263 & $.513^{* *}$ & & 28.921 \\
\hline MCCB-AS $^{3}$ & .177 & $.421^{* *}$ & & 17.456 \\
\hline MCCBVerL $^{4}$ & .138 & $.372^{* *}$ & & 12.846 \\
\hline MCCBVisL $^{5}$ & .108 & $.329^{*}$ & .001 & .141 \\
\hline $\begin{array}{l}\text { WAIS-VCI+WAIS-PRI+MCCB- } \\
\text { AS+MCCBverL+MCCBVisL }\end{array}$ & .312 & $.232 ; 335^{*} ; .024 ; 179 ; 045$ & & \\
\hline
\end{tabular}

${ }^{1}$ Wechsler Adult Intelligence Scale (WAIS) Verbal Comprehension Ind ${ }^{1} \mathrm{ex},{ }^{2}$ WAIS Perceptual

Reasoning Index, ${ }^{3}$ Matrics Consensus Cognitive Battery (MCCB) composite measure of attention and speed, ${ }^{4}$ MCCB Verbal Learning, ${ }^{5} \mathrm{MCCB}$ Visual Learning, ${ }^{*} \mathrm{p}<.05, * * \mathrm{p}=<.001$ 